\title{
Bandwidth Exchange: An Energy Conserving Incentive Mechanism for Cooperation
}

\author{
Dan Zhang, Ryoichi Shinkuma, Member, IEEE, and Narayan B. Mandayam, Fellow, IEEE
}

\begin{abstract}
Cooperative forwarding in wireless networks has shown to yield rate and diversity gains, but it incurs energy costs borne by the cooperating nodes. In this paper we consider an incentive mechanism called Bandwidth Exchange $(B E)$ where the nodes flexibly exchange the transmission bandwidth as a means of providing incentive for forwarding data, without increasing either the total bandwidth required or the total transmit power. The advent of cognitive radios and multicarrier systems such as Orthogonal Frequency Division Multiple Access (OFDMA) with the ability to flexibly delegate and employ a number of subcarriers makes this approach particularly appealing compared to other incentive mechanisms that are often based on abstract notions of credit and shared understanding of worth. We consider a $N$-node wireless network over a fading channel and use a Nash Bargaining Solution (NBS) mechanism to study the benefits of $B E$ in terms of rate and coverage gains. We also propose two heuristic algorithms based on simple probabilistic rules for forwarding and study the tradeoffs in terms of performance among these approaches. Our results reveal that bandwidth exchange based forwarding can provide transmit power savings in OFDMA networks of at least $3 \mathrm{~dB}$ compared to noncooperation.
\end{abstract}

Index Terms-Cognitive radio, incentive mechanism, bandwidth exchange, Nash bargaining, OFDMA.

\section{INTRODUCTION}

C OOPERATIVE forwarding is an essential technique to enhance connectivity and throughput for wireless networks. However, forwarding always incurs some sort of cost - a real cost like power, and/or an opportunity cost like delay. Recent work in [1] has shown that even in the absence of such costs, cooperation among nodes in a wireless network is not guaranteed and may require incentives. Current studies on cooperative forwarding mechanisms largely fall into four categories: reputation based mechanisms [2]-[6], credit based incentives [7], [8], network assisted pricing mechanisms [9], [10] and mechanisms based on forwarding games [11][14]. These prior techniques often mimic the operation of a complex economy and their efficient operation requires

Manuscript received August 20, 2009; revised January 19, 2010; accepted March 27, 2010. The associate editor coordinating the review of this paper and approving it for publication was C. Xiao.

D. Zhang and N. B. Mandayam are with WINLAB, Department of Electrical and Computer Engineering, Rutgers University, Piscataway, NJ, 08854 USA (e-mail: \{bacholic, narayan\}@winlab.rutgers.edu).

R. Shinkuma is with the Graduate School of Informatics, Kyoto University Yoshida-honmachi, Sakyo-ku, Kyoto, 606-8501, Japan (e-mail: shinkuma@i.kyoto-u.ac.jp). He was a visiting scholar at WINLAB, Rutgers University, from Sep. 2008 to Aug. 2009.

This paper has been submitted in part for presentation at IEEE PIMRC 2009.

This work is supported in part by the NSF (no. CCF-0634973 and CNS0721826). It is also supported in part by the Japan Society for the Promotion of Science (JSPS) under Grant-in-Aid for Encouragement of Young Scientists (B) (no. 21760288).

Digital Object Identifier 10.1109/TWC.2010.06.091263 such enablers as a stable currency, a system of credit or a shared understanding of what things are worth. In real economies, these enablers are achieved over long periods of time, and even with experience, the overall functioning of such economies is difficult to predict, a lesson we have learned frequently and with some pain. The main contribution of this paper is to circumvent some of these difficulties by exploring the incentive induced from exchanging a fraction of individually preassigned bandwidth among nodes, referred to as Bandwidth Exchange $(B E)$. Specifically, whenever a node asks another node for cooperative forwarding, it delegates a portion of its frequency resource to the forwarder as immediate compensation for the forwarder's loss. As will be shown shortly, compensation with bandwidth is advantageous over power, especially when the bandwidth available to each node is relatively scarce. This property also makes $B E$ an important and attractive incentive mechanism for forwarding.

Our incentive design in a $N$-node network is based on the two-node Nash Bargaining Solution $(N B S)$ with $B E$, the details of which can be found in [15]. A similar NBS-based cooperation strategy for a two-node network was also discussed in [16]. Recent advances in cognitive radio and multicarrier systems such as OFDMA [17] provide a way to naturally implement this incentive mechanism. In particular, the OFDMA technology currently employed in Mobile WiMAX [18] and LTE [19] allows nodes to flexibly acquire and relinquish a number of the subcarriers, making this mechanism a possible candidate for implementation.

The rest of the paper is organized as follows. In Section II, we motivate $B E$ and present a system model to study it. We describe the details of $B E$ as well as two heuristic algorithms in Section III and a distributed implementation of $B E$ in Section IV. We present the numerical results in Section $\mathrm{V}$ and conclude in Section VI.

\section{System Model AND Bandwidth Exchange}

To motivate $B E$, consider Shannon's canonical channel capacity formula for an AWGN channel with a noise power spectral density of $N_{0} / 2$

$$
C=W \log _{2}\left(1+\frac{P^{\mathrm{t}}}{N_{0} W}\right) .
$$

It is clear that $C$ is only logarithmically dependent on transmit power $P^{\mathrm{t}}$, but nearly linearly dependent on bandwidth $W$, especially when $W$ is relatively small. The largest partial derivatives with respect to these variables are given as

$$
\left.\frac{\partial C}{\partial P^{\mathrm{t}}}\right|_{P^{\mathrm{t}=0}}=\frac{1}{N_{0} \log 2},\left.\quad \frac{\partial C}{\partial W}\right|_{W=0}=\infty .
$$




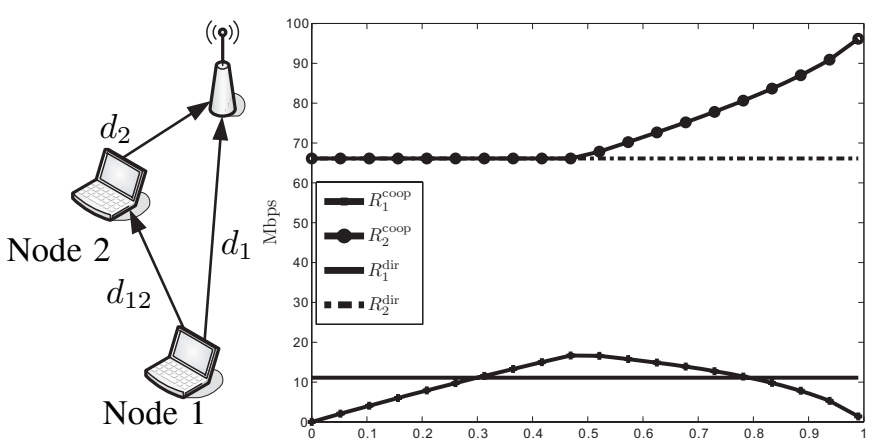

Fig. 1. Bandwidth exchange enhances rates for both nodes simultaneously with $d_{1}=400 \mathrm{~m}, d_{2}=150 \mathrm{~m}, d_{12}=300 \mathrm{~m}, \kappa=6 \times 10^{6} \mathrm{MHz} \cdot \mathrm{m}^{3} / \mathrm{mW}$.

Equation (2) suggests that incentivising forwarding with additional bandwidth seems more promising than using additional transmit power. However, one may question whether it is really beneficial to reallocate bandwidth, since, after all, when one node acquires some bandwidth, the other node loses the same amount of bandwidth. Further, as the bandwidth increases,

$$
\lim _{W \rightarrow \infty} C=\frac{P^{\mathrm{t}}}{N_{0} \log 2}
$$

suggesting that the marginal increase in capacity saturates. A simple example can be constructed to show that this is not a problem when $W$ is small. Consider the two-node network shown in Fig. 1. Suppose each node has a nonoverlapping bandwidth $\left(W_{1}=W_{2}=20 \mathrm{MHz}\right)$ and fixed transmit power $\left(P_{1}^{\mathrm{t}}=P_{2}^{\mathrm{t}}=20 \mathrm{dBm}\right)$. Also suppose that the channels between the access point $(A P)$ and the nodes as well as the channels between the nodes are determined by distance-based path loss, i.e., the rate achieved on a link is an explicit function of the bandwidth $W$ and link gain $\rho$, which is parameterized by its fixed transmit power $P^{\mathrm{t}}$. We assume this function is given by

$$
C=C(W, \rho)=W \log _{2}\left(1+\frac{\rho P^{\mathrm{t}}}{W}\right)
$$

where $\rho=\kappa d^{-3}$ with $d$ being the distance and $\kappa$ being a proportionality constant that also captures the noise power spectral density $N_{0} / 2$. For the specific geometry shown in Fig. 1, it follows that if both nodes only use direct links for transmission, node 1 achieves a transmission rate of $R_{1}^{\text {dir }}=11$ Mbps, while node 2 achieves $R_{2}^{\text {dir }}=66$ Mbps. However, if node 1 chooses to use node 2 as a forwarder and delegates a fraction $x$ of its bandwidth to node 2, then the rates achieved through cooperation are given as

$$
\begin{aligned}
& R_{1}^{\text {coop }}=\min \left\{C_{1}\left((1-x) W_{1}, \rho_{12}\right),\right. \\
& \left.C_{2}\left(W_{2}+x W_{1}, \rho_{20}\right)-R_{2}^{\text {dir }}\right\}, \\
& R_{2}^{\text {coop }}=C_{2}\left(W_{2}+x W_{1}, \rho_{20}\right)-R_{1}^{\text {coop }},
\end{aligned}
$$

where $\rho_{12}$ and $\rho_{20}$ are the link gains from node 1 to node 2 and from node 2 to the $A P$. The functions $C_{1}, C_{2}$ are as defined in (4) and we have assumed that node 2 requires its own rate to be at least $R_{2}^{\text {dir }}$ or better. As shown in the figure, we observe that there is a range of values of $x$ for which both nodes' rates are improved. While we have motivated the power of $B E$ via this simple example, in the rest of the paper



Fig. 2. When the direct link is under outage, node $i$ tries to incent forwarding by delegating $\Delta W_{i j}$ to node $j$.

our focus will be on studying the incentive mechanism in an $N$-node network over fading channels.

Consider $N$ nodes (labeled $1,2, \ldots, N$ ) transmitting to an $A P$ (labeled as node 0). Each node either transmits through the direct link or at most one forwarder, as shown in Fig. 2. Nodes have designated nonoverlapping bandwidths $W_{i}$, fixed transmit power $P_{i}^{\mathrm{t}}$ and minimum required ${ }^{1}$ rates $R_{i}^{\min }$. In what follows, subscript $i j$ always implies the direction from $i$ to $j$. If such a subscript is used in a transmission scheme, it is understood that $i$ is the source and $j$ is the forwarder (or the $A P$ if $j=0$ ). We assume a fading channel model where the transmission is slotted. The link gain $\rho_{i j}\left(=\rho_{j i}\right)$ in each slot is considered static and is the realization of an i.i.d. random variable.

Let $C_{i}^{\text {ins }}(W, \rho)$ denote the instantaneous capacity of some link originating from node $i$ in a slot, given node $i$ 's available bandwidth $W$ and the instantaneous link gain $\rho$. Let $R_{i}^{\text {ins }}$ denote the instantaneous rate of node $i$ in a slot and we assume that it is equal to $C_{i}^{\mathrm{ins}}(W, \rho)$. At the beginning of every slot, node $i$ first attempts to transmit directly to the $A P$, i.e., $R_{i}^{\text {ins }}=C_{i}^{\text {ins }}\left(W_{i}, \rho_{i 0}\right)$. If the direct link $i 0$ is under outage, i.e., $C_{i}^{\text {ins }}\left(W_{i}, \rho_{i 0}\right)<R_{i}^{\text {min }}$, it broadcasts a cooperation request to its neighbors, one of which could help forward node $i$ 's data to $A P$, by means of $B E$. In the course of cooperation between a source node $i$ and a forwarder $j$, node $i$ delegates its available bandwidth up to $W_{i}$ as dictated by $B E$ to node $j$, which forwards its own data as well as the data from node $i$ to the $A P$ with the increased bandwidth available to it. We assume there is no flow splitting and every forwarder serves at most one source. If $i$ cannot find a neighbor to provide such cooperation, $i$ stays under outage for the slot.

The basic idea of cooperation through $B E$ is the source delegating as much of its frequency resource as possible to the forwarder in exchange for cooperation that guarantees the source's minimum required rate. Therefore, when node $j$ forwards for node $i$ through $B E$, node $i$ can withhold

\footnotetext{
${ }^{1}$ While the rate threshold implicitly assumes that the traffic is inelastic, the $B E$ based forwarding idea presented here is more general and also applies to the case of elastic traffic (the example in Fig. 1 assumes no $R_{\min }$ ).
} 
$W_{i}-\Delta W_{i j}$ and delegate $\Delta W_{i j}$ to node $j$ such that

$$
R_{i}^{\mathrm{ins}}=R_{i}^{\mathrm{min}}=C_{i}^{\mathrm{ins}}\left(W_{i}-\Delta W_{i j}, \rho_{i j}\right),
$$

since $i$ only seeks to maintain a connection rate of $R_{i}^{\min }$ to the $A P$. In the mean time, in addition to guaranteeing $R_{i}^{\min }$ for node $i$, node $j$ uses the remaining capacity achieved with increased bandwidth $W_{j}+\Delta W_{i j}$ for its own data,

$$
R_{j}^{\text {ins }}=C_{j}^{\text {ins }}\left(W_{j}+\Delta W_{i j}, \rho_{j 0}\right)-R_{i}^{\min } .
$$

This procedure is illustrated in Fig. 2.

Should cooperation occur between source $i$ and forwarder $j$, equations (5) and (6) define how $B E$ works in this particular setting. Note that they also describe the relationship of the rates and delegated bandwidth $\Delta W_{i j}$ to the link gain $\rho_{i j}$. However, we say the request from node $i$ is not supportable at its neighbor $j$ if either

$$
C_{i}^{\text {ins }}\left(W_{i}, \rho_{i j}\right)<R_{i}^{\min } \quad \text { or } \quad R_{j}^{\text {ins }}<R_{j}^{\min } .
$$

The first condition implies the link $i j$ is so bad that there is no way node $i$ can send at rate $R_{i}^{\min }$ to node $j$. The second condition implies that cooperation with node $i$ will effectively put node $j$ under outage, which includes as a special case that node $j$ itself is looking for cooperation. In either case, node $j$ will definitely refuse to provide cooperative forwarding. In a practical implementation, however, the bandwidth can only be transferred as an integral multiple of certain granularity. This requirement has a nice correspondence to the subcarriers used in a multicarrier system. Exchanging bandwidth is realized by exchanging subcarriers individually owned by or assigned to the nodes. One way to achieve this is to approximate $\Delta W_{i j}$ with a number of subcarriers. When the subcarrier spacing is small, which is often the case since this is one of the design objectives of a multicarrier system, the round-off errors will be negligible.

\section{III. $B E$-BASED ForWARDING IN FADING CHANNELS}

In a fading environment, the role of a node as a forwarder or source can change from slot to slot. Therefore the decision made in a slot should take the consequences it entails in future slots into consideration. This situation is better modeled with an infinitely repeated game [20] [15] with each slot corresponding to a stage game. If node $i$ under outage in a slot requests for cooperation from a potential forwarder $j$ through $B E, j$ has to choose a decision from a binary strategy space, i.e., to cooperate or not. We say node $j$ will make a trivial decision to simply reject cooperation if the request is not supportable. Otherwise node $j$ will choose to cooperate with a probability as will be discussed shortly.

The utility function $u_{j}^{\text {ins }}$ of a stage game for an arbitrary node $j$, called instantaneous rate gain, is defined to be the rate increase achieved in that slot compared to noncooperation. Instantaneous rate gain is closely related to the strategy a node takes. If source $i$ successfully secures cooperation from forwarder $j$, then we have

$$
u_{j}^{\mathrm{ins}}=R_{j}^{\mathrm{ins}}-C_{j}^{\mathrm{ins}}\left(W_{j}, \rho_{j 0}\right), \quad u_{i}^{\mathrm{ins}}=R_{i}^{\mathrm{min}},
$$

where $R_{j}^{\text {ins }}$ is calculated from equation (6). If a node $i$ is not involved in any cooperation either as a source or a forwarder, then $u_{i}^{\text {ins }}=0$. There are two cases in which a node $i$ has zero instantaneous rate gain:

1) as a potential source, node $i$ 's request turns out to be unsupportable at every neighbor;

2 ) as a potential forwarder, node $i$ does not receive any supportable request.

If either case is true, we say this stage game (i.e., this slot) is trivial to node $i$. We model the utility function of the repeated game for an arbitrary node $j$ as the average rate gain. From the previous discussion, the average rate gain for node $i$ is given by ${ }^{2}$

$$
E\left[u_{i}^{\text {ins }}\right]=\left(1-P_{i}^{\text {trivial }}\right) E\left[u_{i}^{\text {ins }} \mid \text { nontrivial stage game }\right] .
$$

Once the probability distribution function of link gains are known, we can calculate the probability $P_{i}^{\text {trivial }}$ with which a stage game becomes trivial for node $i$. Therefore we only need to focus on nontrivial stage games and disregard those stage games that are trivial to $i$. In other words, for node $i$ we only consider those stage games in which either $i$ is a source and sends a supportable request to some node $j$, or $i$ is a potential forwarder and receives at least one supportable request from some source node. As a consequence, rather than the average rate gain, we define the utility function of the repeated game for node $i$ as the average rate gain conditioned on a nontrivial stage game, i.e.,

$$
u_{i}=E\left[u_{i}^{\text {ins }} \mid \text { nontrivial stage game }\right] .
$$

Note in this definition, $u_{i}$ is not only dependent on channel statistics, but also on the strategy node $i$ takes in deciding whether to forward for other nodes.

\section{A. The Two-Node NBS Revisited}

Suppose we have a two-node network consisting of node $i$ and node $j$. As discussed before, we overlook the trivial stage game for node $i$, or equivalently, the trivial stage game for node $j$ since we only have two nodes. In any given nontrivial stage game, with probability $P_{i j}$ node $i$ sends a request to node $j$ for cooperation and with probability $P_{j i}=1-P_{i j}$ the request goes the other way around. If node $j$ forwards data for node $i$, we use $u_{i j}^{\mathrm{f} \text {, ins }}$ and $u_{i j}^{\mathrm{s} \text {, ins }}$ to denote the instantaneous rate gain of the forwarder node $j$ and the source node $i$, respectively. Correspondingly, their averages are denoted as $u_{i j}^{\mathrm{f}}$ and $u_{i j}^{\mathrm{s}}$. It follows from (8) and (9) that

$$
\begin{aligned}
u_{i j}^{\mathrm{f}} & =E\left[R_{j}^{\mathrm{ins}}-C_{j}^{\mathrm{ins}}\left(W_{j}, \rho_{j 0}\right) \mid \text { nontrivial stage game }\right], \\
u_{i j}^{\mathrm{s}} & =R_{i}^{\mathrm{min}} .
\end{aligned}
$$

An extensive form of the nontrivial part of the repeated game is shown in Fig. 3. Note when the potential forwarder chooses not to cooperate, the average rate gains for both nodes are zero while in case of cooperation the forwarder's expected rate can be negative.

The normal form of the game, as shown in Table I, consists of four strategy profiles and their associated payoff profiles, denoted by $\langle\mathrm{n}, \mathrm{c}\rangle,\langle\mathrm{c}, \mathrm{c}\rangle,\langle\mathrm{c}, \mathrm{n}\rangle$ and $\langle\mathrm{n}, \mathrm{n}\rangle$, where $\langle\mathrm{n}, \mathrm{c}\rangle$ (abbreviation for $\langle$ noncooperation, cooperation $\rangle$ ) means node $j$

\footnotetext{
${ }^{2}$ In this article, expectations are all taken over the random link gains across slots.
} 


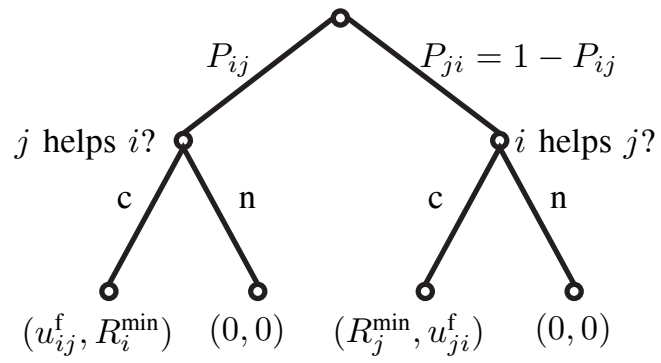

Fig. 3. Extensive form of the two-node stage game.

TABLE I

NORMAL FORM OF THE STAGE GAME.

\begin{tabular}{|c|c|c|}
\hline$j$ & cooperation (c) & noncooperation (n) \\
\hline $\mathrm{c}$ & $\begin{array}{c}\left(P_{j i} R_{j}^{\min }+P_{i j} u_{i j}^{\mathrm{f}},\right. \\
\left.P_{i j} R_{i}^{\min }+P_{j i} u_{j i}^{\mathrm{f}}\right)\end{array}$ & $\left(P_{i j} u_{i j}^{\mathrm{f}}, P_{i j} R_{i}^{\min }\right)$ \\
\hline $\mathrm{n}$ & $\left(P_{j i} R_{j}^{\min }, P_{j i} u_{j i}^{\mathrm{f}}\right)$ & $(0,0)$ \\
\hline
\end{tabular}

would choose not to forward for $i$ if $i$ requests its cooperation while $i$ would choose to forward for $j$ if $j$ requests its cooperation. Similar interpretations apply for the other strategy profiles. Based on the chosen strategies, $u_{j}$ and $u_{i}$ defined in (9) form a plane, on which we let the points $D, E, F, O$ denote the payoff profiles associated with the strategy profiles $\langle\mathrm{n}, \mathrm{c}\rangle,\langle\mathrm{c}, \mathrm{c}\rangle,\langle\mathrm{c}, \mathrm{n}\rangle$ and $\langle\mathrm{n}, \mathrm{n}\rangle$, respectively. Coordinates of these points are calculated by following different paths on the extensive form conditioned with probabilities $P_{i j}$ and $P_{j i}$. For example, the coordinates of $D$ are given as

$$
D=P_{i j} \cdot(0,0)+P_{j i} \cdot\left(R_{j}^{\min }, u_{j i}^{\mathrm{f}}\right)=\left(P_{j i} R_{j}^{\min }, P_{j i} u_{j i}^{\mathrm{f}}\right) .
$$

Coordinates of $E, F, O$ are calculated similarly and are given as

$$
\begin{aligned}
& E=\left(P_{j i} R_{j}^{\mathrm{min}}+P_{i j} u_{i j}^{\mathrm{f}}, P_{i j} R_{i}^{\mathrm{min}}+P_{j i} u_{j i}^{\mathrm{f}}\right), \\
& F=\left(P_{i j} u_{i j}^{\mathrm{f}}, P_{i j} R_{i}^{\mathrm{min}}\right), \\
& O=(0,0) .
\end{aligned}
$$

The convex hull $\mathcal{C}$ of the four points is a parallelogram (see Appendix) and defines the feasible region of payoff profiles as shown in Fig. 4. Each point $\left(u_{j}, u_{i}\right) \in \mathcal{C}$ represents a set of payoff profiles achievable by mixing $\langle\mathrm{n}, \mathrm{c}\rangle,\langle\mathrm{c}, \mathrm{c}\rangle,\langle\mathrm{c}, \mathrm{n}\rangle$ and $\langle\mathrm{n}, \mathrm{n}\rangle$ with corresponding probabilities $\lambda_{1}, \lambda_{2}, \lambda_{3}, \lambda_{4}$. The two-node $N B S$ is a point $S=\left(u_{j}, u_{i}\right) \in \mathcal{C}$ such that the proportional fairness metric of the two average rate gains is maximized, i.e.,

$$
\begin{array}{ll}
\underset{\lambda_{1}, \lambda_{2}, \lambda_{3}, \lambda_{4}}{\max } & u_{i} u_{j} \\
\text { s.t. } & u_{j}=\lambda_{1} P_{j i} R_{j}^{\mathrm{min}}+\lambda_{2}\left(P_{j i} R_{j}^{\min }\right. \\
& \left.+P_{i j} u_{i j}^{\mathrm{f}}\right)+\lambda_{3} P_{i j} u_{i j}^{\mathrm{f}}+\lambda_{4} \cdot 0, \\
& u_{i}=\lambda_{1} P_{j i} u_{j i}^{\mathrm{f}}+\lambda_{2}\left(P_{i j} R_{i}^{\min }\right. \\
& \left.+P_{j i} u_{j i}^{\mathrm{f}}\right)+\lambda_{3} P_{i j} R_{i}^{\min }+\lambda_{4} \cdot 0, \\
& \lambda_{1}+\lambda_{2}+\lambda_{3}+\lambda_{4}=1, \\
& \lambda_{i} \geq 0, \quad i=1,2,3,4 .
\end{array}
$$

Once the optimal mixing probabilities $\lambda_{i}$ are obtained, the cooperation probability $P_{i j}^{\mathrm{c}}$ of node $j$ when it receives a



Fig. 4. Feasible region and $N B S$ on the pareto frontier.

supportable request from node $i$ is given by

$$
\begin{aligned}
P_{i j}^{\mathrm{c}} & =\operatorname{Prob}(j \text { takes strategy } \mathrm{c}) \\
& =\operatorname{Prob}((j, i) \text { take }\langle\mathrm{c}, \mathrm{c}\rangle)+\operatorname{Prob}((j, i) \text { take }\langle\mathrm{c}, \mathrm{n}\rangle) \\
& =\lambda_{2}+\lambda_{3} .
\end{aligned}
$$

For $P_{j i}^{\mathrm{c}}$ with similar definition, we have $P_{j i}^{\mathrm{c}}=\lambda_{1}+\lambda_{2}$.

For the two-node $N B S$, a geometric interpertation exists [15] [21] for the solution of (10). The solution is given by $S$ in Fig. 4 where the slope of the line segment $O S$ is the negative slope of the subgradient of $\mathcal{C}$ at $S$. Use subscript $x$ and $y$ to denote the horizontal and vertical coordinates of a point, so $D=\left(D_{x}, D_{y}\right), E=\left(E_{x}, E_{y}\right), F=\left(F_{x}, F_{y}\right)$. Define $\tan D=D_{y} / D_{x}$ and define $\tan E, \tan F$ similarly. Then, we can derive the cooperation probability $P_{i j}^{\mathrm{c}}$ explicitly as in (12) (see Appendix). The formula for $P_{j i}^{\mathrm{c}}$ is symmetric with subscripts transposed.

\section{B. Pairwise N-Node Bargaining}

The precise $N$-node $N B S$ also seeks to yield proportionally fair average rate gains for all $N$ nodes with mixed strategy profiles. However, it is practically infeasible to formulate if $N$ is large, simply because the strategy space for each node grows exponentially as the number of nodes in the network increases. This prompts us to look for suboptimal solutions with much lower complexity. One such solution is based on restricting cooperation to two-hop forwarding. Since we also required that one forwarder for one source and no flow splitting, eventually cooperation happens only between disjoint pairs of nodes, each pair consisting of a source and a forwarder. It is then natural to approximate the $N$-node bargaining with a series of two-node bargainings as derived in III-A, which we call the pairwise $N$-node bargaining or simply pairwise bargaining. Pairwise bargaining achieves huge reduction in complexity by ignoring the interaction between different pairs - with pairwise bargaining, a node considers itself under outage if the direct link is out, but in fact it is under outage only if it does not successfully secure any cooperation either. 


$$
P_{i j}^{\mathrm{c}}= \begin{cases}0, & \tan D>|\tan F|, \\ -\frac{P_{j i}}{2 P_{i j}}\left(\frac{R_{j}^{\mathrm{min}}}{u_{i j}^{\mathrm{f}}}+\frac{u_{j i}^{\mathrm{f}}}{R_{i}^{\mathrm{min}}}\right), & |\tan E|>|\tan F|>|\tan D|, \\ 1, & \text { otherwise. }\end{cases}
$$

\section{Selection Policies}

Pairwise bargaining in a $N$-node network implies that each forwarder may have to select one from many sources to cooperate with, while each source select one from many forwarders. Thus we must address how a forwarder determines which request to be granted and how a source determines which cooperating forwarder to follow. Both issues are called selection policies.

To be more specific, in pairwise bargaining $P_{i j}^{\mathrm{c}}$ calculated from equation (12) should not be taken directly as the probability that forwarder $j$ offers cooperation to source $i$ since $i$ could be simply one of the supportable sources for which a cooperation probability is calculated using the two-node $N B S$ solution. Instead, all such sources are put in a candidate list $\mathcal{L}_{f}$ with an individual probability of $P_{i j}^{\mathrm{c}}$. After $\mathcal{L}_{f}$ is compiled, the forwarder side selection policy is invoked to pick a source to really cooperate with. Because each candidate had an independent bargaining with node $j$ and was put in $\mathcal{L}_{f}$ according to the cooperation probability calculated from the bargaining solution in (12), we require that node $j$ pick one of them randomly to ensure fairness.

The source also compiles a list $\mathcal{L}_{s}$ of candidate forwarders. It then picks a forwarder from it to follow by the source side selection policy if $\mathcal{L}_{s}$ is not empty. Inspired by (10), the source side selection policy seeks to maximize the product of instantaneous source-forwarder rate gains. A source node $i$ would have no "disincentive" for this choice because with any cooperating forwarder $j, i$ always has $u_{i j}^{\mathrm{s} \text {, ins }}=R_{i}^{\mathrm{min}}$, a fixed value. Consequently, the selection policy turns out to be picking the forwarder $j$ with the maximum instantaneous rate gain from $\mathcal{L}_{s}$, i.e.,

$$
j=\underset{k}{\arg \max }\left\{u_{i k}^{\mathrm{f}, \text { ins }}\right\}
$$

\section{Simple Heuristic Algorithms}

Although NBS performs desirably in many aspects as to be shown later, the complexity of solving (10) can be too high for some applications. For this reason, we also propose two simple heuristic algorithms that employ $B E$. These algorithms reach a decision based on instantaneous observations and do not require parameter estimation, eliminating the overhead of corresponding message exchange. These algorithms will either suffer severe unfairness or degraded performance as to be shown later by simulation. However, they serve as good bench marks for performance as well as a nice tradeoff when reduced complexity or network overhead is a bigger concern.

1) Myopic Strategy: The myopic strategy $(M S)$ is one where a node refuses to forward unless forwarding is a guaranteed advantage to take. Assuming $B E$ is still employed, a myopic forwarder $j$ will set $P_{i j}^{\mathrm{c}}=1$ and put it on a candidate source list only if $u_{i j}^{\mathrm{f} \text {,ins }}>0$. If the candidate source list is not empty, the forwarder would exercise the myopic forwarder side selection policy by selecting the source $i$ such that

$$
i=\underset{k}{\arg \max }\left\{u_{k j}^{\mathrm{f} \text {,ins }}\right\} \text {. }
$$

Because every cooperating forwarder guarantees the same minimum required rate for a source, the myopic source side selection policy would randomly pick a forwarder to follow, if there is any. Note without $B E, M S$ induces no cooperation.

2) Altruistic Strategy: The altruistic strategy $(A S)$, as suggested by its name, represents a very generous type of cooperation strategy. Assuming $B E$ is still employed, an altruistic forwarder $j$ will cooperate with a source $i$ as long as the request is supportable (see (7)) by putting it on a candidate list. If the candidate source list is not empty, $j$ would exercise the altruistic forwarder side selection policy by randomly picking one to cooperate with. The altruistic source side selection policy would be to pick the forwarder that would benefit most from cooperation, i.e., using equation (13). Note without $B E$, $A S$ has no effect on network throughput improvement, but it does reduce the outage probability of nodes at the price that some forwarders' average data rates will also be reduced, due to its over generosity.

\section{Distributed Algorithm Design for $B E$}

Pairwise bargaining requires a certain amount of message exchange between the source nodes and the forwarder nodes. In addition to sending the updated estimates back to the source nodes, the forwarder needs to send an acknowledgement to the source that it decides to cooperate with. Similarly, a source node receiving an acknowledgement of cooperation will make a decision whether to accept the offer and sends an acknowledgement back to the forwarder before it proceeds to data transmission. In this section, we will present distributed algorithms of $B E$ with $N B S$ as well as $M S$ and $A S$. There is a critical issue that needs to be addressed before we give the algorithm of $B E$ with $N B S$, i.e., estimation of all the necessary parameters for solving the problem in (10).

\section{A. Parameter Estimation}

To solve equation (10), a node needs to know a few parameters including $P_{i j}, P_{j i}, u_{i j}^{\mathrm{f}}$ and $u_{j i}^{\mathrm{f}}$, through estimation. In particular, a forwarder needs these parameters to calculate its decision. Thus these parameters are estimated at the forwarder side and are communicated to the source side by message exchange. For $B E$ with $N B S$, larger network overhead is incurred for this purpose compared to $M S$ and $A S . R_{i}^{\min }$ and $R_{j}^{\mathrm{min}}$ are prescribed parameters, which can be exchanged through messages once and for all. This is common to both the $N B S$ based and heuristic algorithms. 


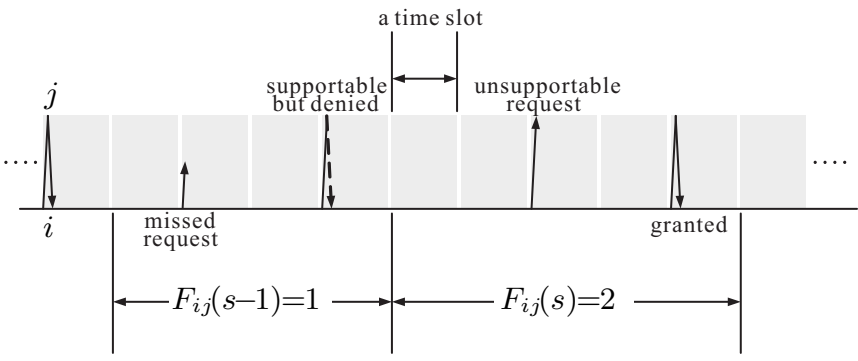

Fig. 5. Estimating $P_{i j}$ by counting the number of slots between two supportable requests from node $i$ to node $j$.

Because we assume channel statistics remain unchanged and channel realizations independent across slots, the best estimates of $P_{i j}$ and $u_{i j}^{\mathrm{f}}$ are obtained by taking the sample means. For example, a new estimate of $u_{i j}^{\mathrm{f}}$ is obtained from $u_{i j}^{\mathrm{f} \text {, ins }}$ every time $j$ receives a supportable request from $i$. The estimate is given by $\hat{u}_{i j}^{\mathrm{f}}(T)=\left(\sum_{t=1}^{T} u_{i j}^{\mathrm{f} \text {, ins }}(t)\right) / T$, where $t=1,2, \ldots, T$ is the index of requests from $i$ to $j$. However, to enable the estimator to track the slow variation of a nonstationary channel, the estimator needs to rely more on recent observations. This is possibly achieved by using a low pass filter $h_{u}(z)=\alpha /\left(1-(1-\alpha) z^{-1}\right)$, where $\alpha>0$ is a small forgetting factor. The estimate is hence given by $\hat{u}_{i j}^{\mathrm{f}}(t)=h_{u}\left(u_{i j}^{\mathrm{f} \text {,ins }}(t)\right)$.

The estimation of $P_{i j}$, the probability that node $j$ receives a supportable request from node $i$, is based on counting the number of slots between two such requests. This idea is shown in Fig. 5. Let $F_{i j}(s)$ be the number of slots between $(s-1)$ th and $s$ th supportable requests from $i$ to $j$, then $1 / F_{i j}(s)$ is an unbiased estimate of $P_{i j}$. The maximum likelihood (ML) estimate of $P_{i j}$ from $s$ such observations is given by $\hat{P}_{i j}=s / \sum_{k=1}^{s} F_{i j}(k)$. Like the estimation of $u_{i j}^{\mathrm{f}}$, to cope with nonstationary channels, a low pass filter is preferred for practical application, i.e., $\hat{P}_{i j}=1 / h_{P}\left(F_{i j}(s)\right)$.

\section{B. Distributed Algorithm for N-Node Pairwise Bargaining}

In this subsection we will give the algorithms for $B E$ with $N B S$. At the beginning of each slot, if a node's direct link is under outage, it automatically becomes a potential source and executes Alg. 1; otherwise it becomes a potential forwarder and executes Alg. 2. All nodes register the variables they calculate or receive from other nodes through messages across slots. We assume $i$ represents a general source and $j$ a general forwarder and give the distributed algorithms for both source and forwarder sides in terms of $i$ and $j$. We use $h$ to denote the filters used in parameter estimation at various places of the algorithm. In practice, these filters can be (and should be) different to suit their respective purposes.

\section{Distributed Algorithms Based on Simple Heuristics}

The distributed algorithms for the source and forwarder nodes based on $M S$ and $A S$ strategies differ from that of $B E$ with $N B S$ in terms of the selection policies employed (see section III-D) and how a forwarder decides which source node to put in the candidate source list and vice versa.
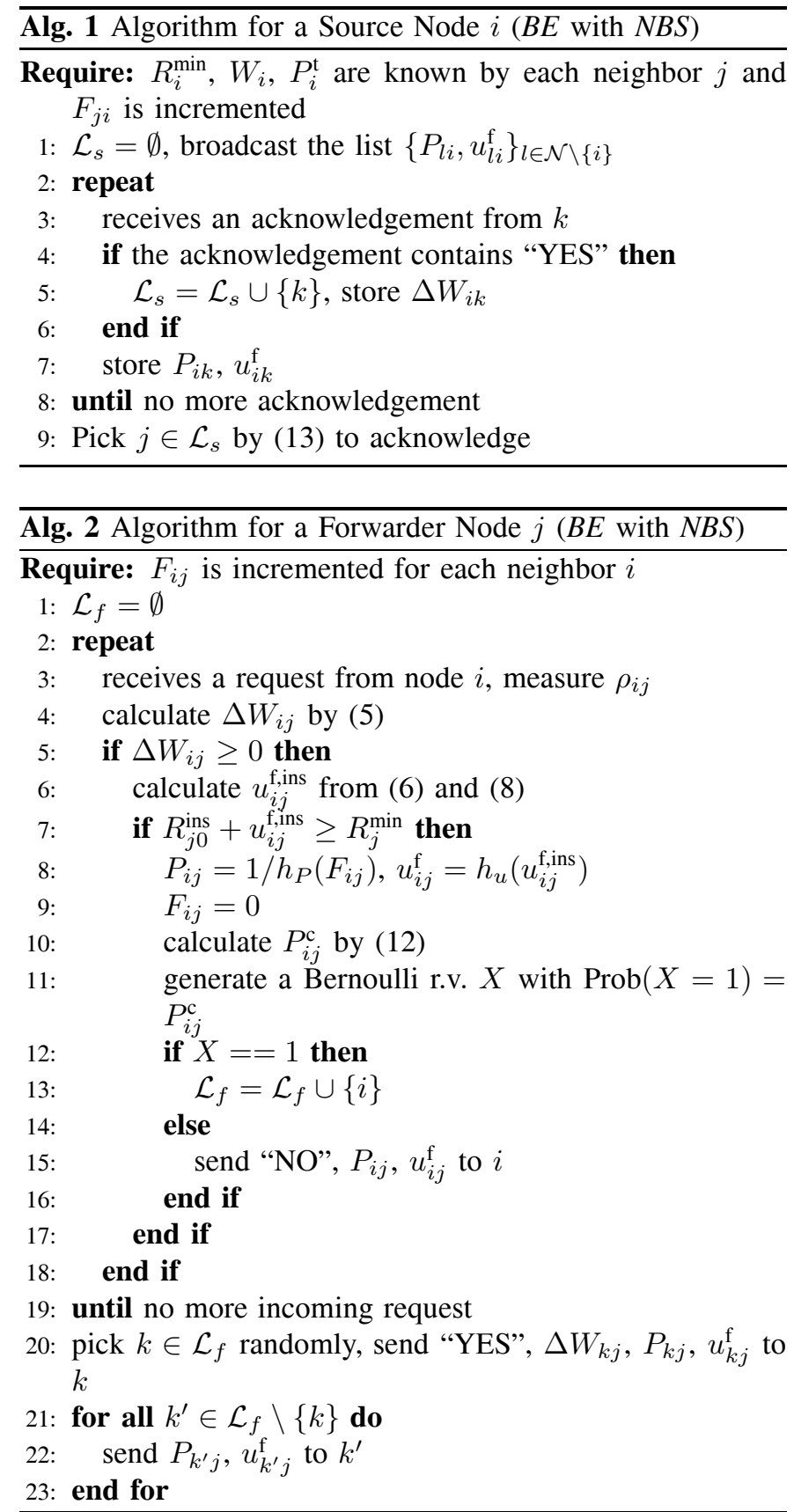

\section{Numerical Results}

\section{A. Simulation model}

For the purpose of illustration, we consider an OFDMA like transmission scheme with parameters much like the one used in mobile WiMAX. The presence of orthogonal subcarriers in an OFDMA system provides a natural platform for implementing $B E$ by exchanging orthogonal frequency bands.

We simulate a slotted system using parameters that are typical to mobile WiMAX. Each node is pre-assigned 20 $\mathrm{dBm}$ fixed transmit power [22] [23] and $500 \mathrm{kHz}$ transmission bandwidth corresponding to 50 subcarriers at $10 \mathrm{kHz}$ spacing. When a node delegates bandwidth, it transfers a number of the subcarriers to a forwarder. Since nodes in our network use mutually orthogonal portions of frequency, we model the instantaneous capacity of link $i j$ using its information- 


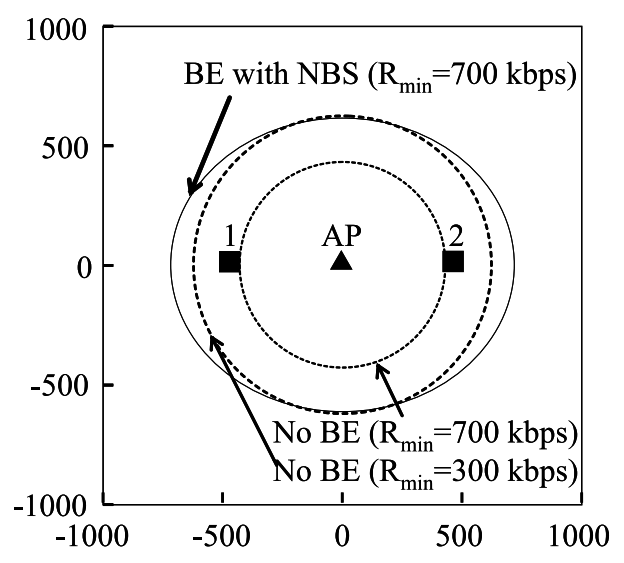

Fig. 6. Improvement in coverage and rate in a $2000 \times 2000 \mathrm{~m}^{2}$ region.

theoretic rate

$$
R_{i j}^{\mathrm{ins}}\left(W, \rho_{i j}\right)=W \log _{2}\left(1+\frac{\rho_{i j} P_{i}^{\mathrm{t}}}{W}\right), i, j=0,1, \ldots, N .
$$

Links are under independent Rayleigh fading and the link gain in each slot is an independent realization of a Rayleigh random variable. Equivalently, this implies that $\rho_{i j}$ is exponentially distributed

$$
p\left(\rho_{i j}\right)=\frac{1}{\bar{\rho}_{i j}} \exp \left(-\frac{\rho_{i j}}{\bar{\rho}_{i j}}\right)
$$

where the statistical mean $\bar{\rho}_{i j}$ is given by the path loss model

$$
\bar{\rho}_{i j}=\kappa d^{-3}, \quad\left(\kappa=6 \times 10^{6} \mathrm{MHz} \cdot \mathrm{m}^{3} / \mathrm{mW}\right) .
$$

The above simulation model implicitly assumes that the average rate of a transmission is one that is obtained when all the subcarriers used undergo identical fading. This is done for the simplicity of illustration but the idea of $B E$ and its applicability to frequency selective OFDMA systems is still valid. The pairwise $N B S$ with $B E$ in (10), as well as $M S$ and $A S$, are implemented for the above channel model. For each simulation we present below, the minimum required rate for every node is $700 \mathrm{kbps}$ unless otherwise specified. We simulate for sufficiently many slots to assess the average performance.

\section{B. A Three-Node Example}

We first present a three-node example to show the power of $B E$ with $N B S$ in improving coverage and rate. Suppose node 1 is fixed at $(-450 \mathrm{~m}, 0)$ and node 2 at $(450 \mathrm{~m}, 0)$. Node 3 is allowed to vary its location in a $2000 \times 2000 \mathrm{~m}^{2}$ region as shown in Fig. 6. The dotted line delineates the area in which the outage probability for node 3 is less than $10 \%$ without cooperation. The solid line delineates the area with improved coverage achieved when using $B E$ with $N B S$ for the same level of outage. The dashed line delineates a comparable coverage area without cooperation. However, the minimum required rate is now lowered to $300 \mathrm{kbps}$ to generate an identical level of outage. This simple illustration indicates that $B E$ can be used to either increase coverage, or increase supported rate.

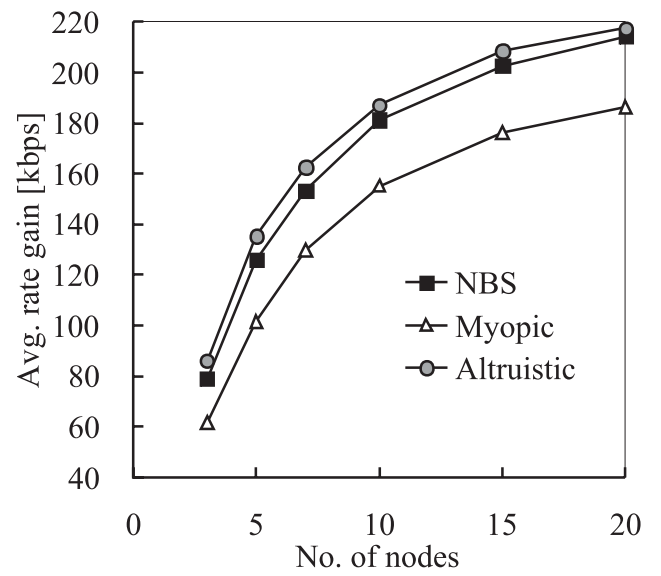

Fig. 7. Average rate gain in a cell consisting of varied number of nodes, minimum required rate $=700 \mathrm{kbps}$.

\section{Comparative Evaluation of Cooperative Forwarding Strategies}

In this section, we present a comparative evaluation of $B E / N B S$ with $M S$ and $A S$. As mentioned earlier, we simulate a slotted system that uses parameters typical to Mobile WiMAX. We consider up to 20 nodes randomly placed in a cell with a radius of $1000 \mathrm{~m}$. Our results are obtained by averaging over multiple time slots and location instantiations of mobiles. We look at the metrics of average rate gain, spectrum efficiency and fairness as a function of the number of nodes in the system and present the corresponding results. In the end, we will present simulation results on power savings.

1) Average Rate Gain: Fig. 7 shows the average rate gain over the rate achieved under no cooperation at all. No matter which algorithm is used, the average rate gain is an increasing function of the number of nodes in the system, illustrating the benefits of user cooperation diversity. $A S$ exhibits the best performance thanks to its generous nature, though nodes close to the $A P$ that serve as the forwarders can suffer a substantial loss in their own rates. $N B S$ performs nearly as good as $A S$ while being fair. These observations will be discussed further when we address fairness. NBS also performs better than $M S$, which represents a very stingy cooperation strategy compared to $A S$. Because nodes are randomly placed in the cell, as the number of nodes increases, eventually any source is almost certain to get cooperation from some forwarder. Therefore all the curves tend to saturate when more nodes are placed in the cell.

2) Spectrum Efficiency: Fig. 8 shows the spectrum efficiency per node averaged over the number of nodes to illustrate the effect of user cooperation diversity. Note that in our model, nodes are employing orthogonal subcarriers and hence do not interfere each other. However, the spectrum efficiency per node increases with the number of nodes. The absence of cooperation diversity, i.e., noncooperation, performs well below the three cooperative strategies. In this example, when the number of nodes is large, $N B S$ performs nearly as well as $A S$ again.

3) Fairness: The NBS does not take average rate gain or spectrum efficiency as an explicit optimization objective. Rather, it provides a proportionally fair rate allocation under 


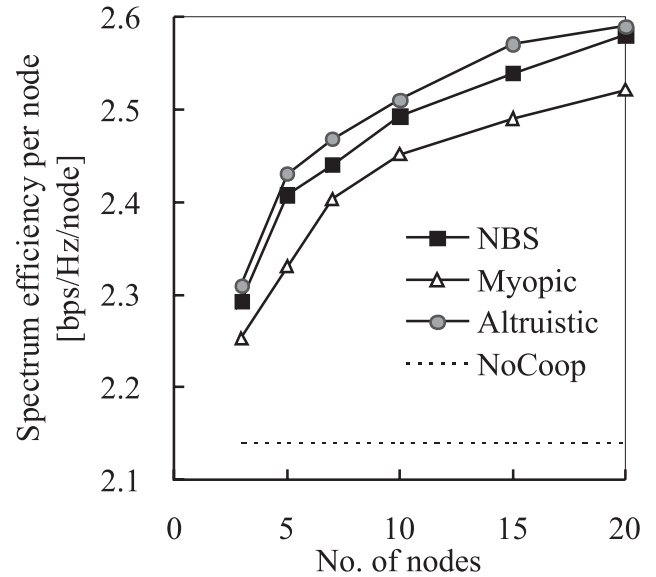

Fig. 8. Spectrum efficiency per node, minimum required rate $=700 \mathrm{kbps}$.

certain constraints, i.e., it tries to maximize the product of rate gains, or equivalently, the geometric mean of rate gains. As a suboptimal solution, the pairwise bargaining strategy does not solve this problem precisely, but a comparison with other strategies in terms of this particular objective would be meaningful. Moreover, the geometric mean of rate gains can be regarded a measure of the average amount of individual incentive that a node has for cooperation no matter what strategy it takes. Let $\mathcal{I}$ denote the geometric mean given as

$$
\mathcal{I}=\left(\prod_{i=1}^{N} \max \left(u_{i}, 0\right)\right)^{1 / N} .
$$

Technically, $\mathcal{I}$ is the geometric mean only if $u_{i}>0$ for all $i$. Otherwise $\mathcal{I}=0$, indicating some nodes receive negative rate gains. In this case, cooperation in fact can not occur because nodes suffering negative rate gains can make a unilateral decision and quit the cooperative system to maintain a rate gain of at least zero. Fig. 9 shows $\mathcal{I}$ as a function of the number of nodes. We observe that $N B S$ performs better than $A S$ almost always by $10 \mathrm{kbps}$, which in turn is better than $M S$ by $20 \mathrm{kbps}$. These numbers can be read as the difference of individual amount of incentive achieved with different strategies. Note that $A S$ due to its over generous nature is inherently unfair. In fact, our experiments reveal that in roughly $10 \%$ of simulation trials, one or more nodes experience negative rate gains. This number increases to $60 \%$ if the minimum required rate for each node is $900 \mathrm{kbps}$.

4) Power Savings: As pointed out at the beginning of the paper, cooperative forwarding improves coverage. The improvement can also be achieved by the traditional noncooperative means at much larger transmit power. In other words, $B E$ based forwarding can be thought of as providing significant transmit power savings for the same level of coverage (outage) experienced by a noncooperative scheme. Fig. 10 shows the power savings of $N B S$ and $M S$ compared to noncooperation, to achieve an average outage probability of 0.1 in various scenarios. Each scenario is parameterized with different number of nodes and minimum required rates. Because the cooperative strategy described by $A S$ is not achievable due to its unfairness as discussed previously, it is not included in the comparison. For the chosen scenarios, the minimum power saving is shown

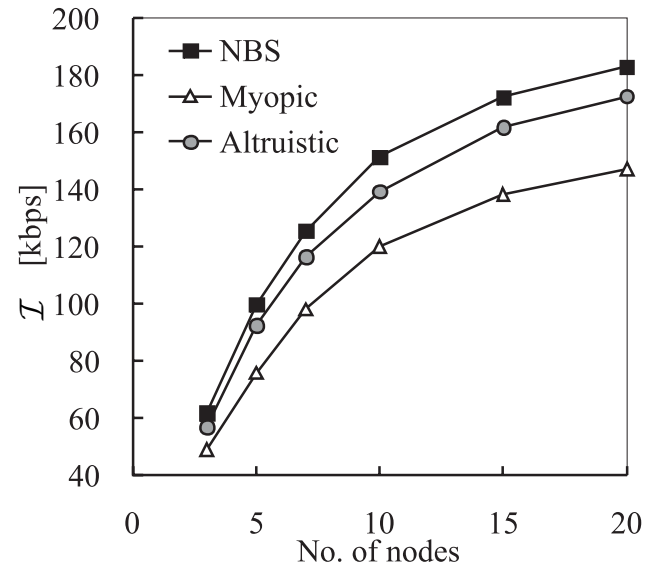

Fig. 9. Geometric mean of rate gains as a measure of fairness, minimum required rate $=700 \mathrm{kbps}$.

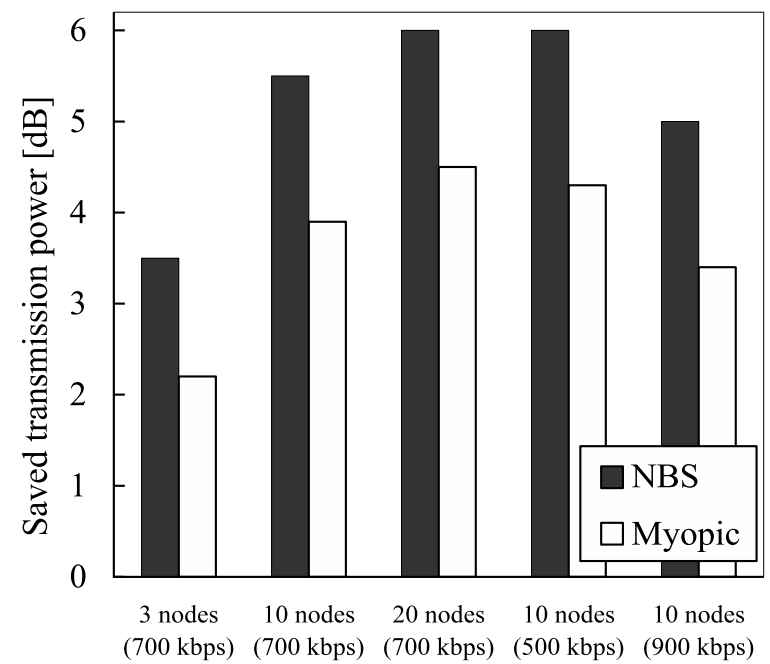

Fig. 10. Power savings of $N B S$ and $M S$ compared to noncooperation, to achieve an outage probability of 0.1 . Horizontal axis shows various scenarios with different number of nodes and minimum required rates.

to be at least $3 \mathrm{~dB}$ for $N B S$ and $2 \mathrm{~dB}$ for $M S$. As the number of the nodes increases, the user cooperation diversity gain increases and therefore the power savings increase to as large as $6 \mathrm{~dB}$ for $N B S$ and $4 \mathrm{~dB}$ for $M S$.

\section{CONCLUSION AND Discussions}

In this paper we discussed a cooperative forwarding incentive mechanism called Bandwidth Exchange where nodes forward data in exchange for bandwidth from the sources. Compared to other incentive mechanisms that are often based on abstract notions of credit and shared understanding of worth, such simple bandwidth delegation provides a more tangible and immediate incentive mechanism. Specifically, we considered a $N$-node wireless network and used a Nash Bargaining Solution to study the benefits of $B E$ in terms of rate and coverage gains. We also proposed two heuristic algorithms based on simple probabilistic rules for forwarding: (1) the Myopic Strategy which admits cooperation only if it incurs a positive rate gain for the forwarder and (2) the Altruistic Strategy which admits cooperation whenever it is supportable. Our results indicated that all $B E$ based schemes 
provided improvements in coverage and rate. Further, the NBS also assured that the rate allocations were proportionally fair. Our results also indicated that wireless networks implementing $B E$ receive significant transmit power savings compared to traditional noncooperative networks. The advent of cognitive radios with the ability to flexibly change their carrier frequency as well as their transmission bandwidth makes the $B E$-based incentive mechanism particularly attractive. Further, the use of OFDMA based access, such as in WiMAX and LTE systems, allows for the flexible exchange of frequency bands among the nodes. The $B E$ idea presented here prompts future investigation into its applicability in a variety of contexts including design of protocols and evaluation of overheads, as well as study of advanced coding and combining techniques for additional performance gains.

\section{APPENDIX}

\section{PROOF OF EQUATION (12)}

The feasible region $\mathcal{C}$ (see Fig. 4) is the convex hull of points $D, E, F, O$ representing the payoff profiles of node $j$ and $i$ achieved with pure strategy profiles $\langle\mathrm{n}, \mathrm{c}\rangle,\langle\mathrm{c}, \mathrm{c}\rangle,\langle\mathrm{c}, \mathrm{n}\rangle$ and $\langle\mathrm{n}, \mathrm{n}\rangle$ respectively. We will prove (12) by exploring the geometric properties of $\mathcal{C}$. As pointed out in [21], the Nash bargaining solution $S$ is a point on the pareto frontier of $\mathcal{C}$ in the first quadrant such that the horizontal image of $O S$ is a subgradient of $\mathcal{C}$ at $S$. This implies $S$ is either on segment $D E$ or segment $E F$, and a necessary condition for the subgradient is that it has a negative derivative. We begin with three propositions which help us classify the possible configurations of the points $D, E, F, O$ (e.g., in which quadrants these points reside).

Proposition 1: $O D E F$ is a parallelogram.

Proof: This is true because

$$
\begin{aligned}
& \overrightarrow{D E}=\overrightarrow{O E}-\overrightarrow{O D} \\
= & \left(P_{j i} R_{j}^{\mathrm{min}}+P_{i j} u_{i j}^{\mathrm{f}}, P_{i j} R_{i}^{\mathrm{min}}+P_{j i} u_{j i}^{\mathrm{f}}\right)-\left(P_{j i} R_{j}^{\mathrm{min}}, P_{j i} u_{j i}^{\mathrm{f}}\right) \\
= & \left(P_{i j} u_{i j}^{\mathrm{f}}, P_{i j} R_{i}^{\mathrm{min}}\right)=\overrightarrow{O F} .
\end{aligned}
$$

Proposition 2: If $u_{i j}^{\mathrm{f}}<0$ or $u_{j i}^{\mathrm{f}}<0$, then $O \rightarrow D \rightarrow$ $E \rightarrow F \rightarrow O$ goes counterclockwise.

Proof: The cross product of $\overrightarrow{O D}$ and $\overrightarrow{O F}$ is perpendicular to the $x-y$ plane, i.e., along the $z$ direction, and is given as

$$
\begin{aligned}
\overrightarrow{O D} \times \overrightarrow{O F} & =\left(P_{j i} R_{j}^{\min }, P_{j i} u_{j i}^{\mathrm{f}}, 0\right) \times\left(P_{i j} u_{i j}^{\mathrm{f}}, P_{i j} R_{i}^{\min }, 0\right) \\
& =\left(0,0, P_{i j} P_{j i}\left(R_{i}^{\min } R_{j}^{\min }-u_{i j}^{\mathrm{f}} u_{j i}^{\mathrm{f}}\right)\right),
\end{aligned}
$$

where we use the triple to denote the magnitudes in the $x, y, z$ directions. If $u_{i j}^{\mathrm{f}} \geq 0>u_{j i}^{\mathrm{f}}$ or $u_{j i}^{\mathrm{f}} \geq 0>u_{i j}^{\mathrm{f}}$, then

$$
R_{i}^{\min } R_{j}^{\min }-u_{i j}^{\mathrm{f}} u_{j i}^{\mathrm{f}}>0 .
$$

If $u_{i j}^{\mathrm{f}}<0$ and $u_{j i}^{\mathrm{f}}<0$, we still have (20). In fact, if $i$ is the source and $j$ is the forwarder, then $C_{j}^{\text {ins }}\left(W_{j}+\Delta W_{i j}, \rho_{j 0}\right)>$ $C_{j}^{\text {ins }}\left(W_{j}, \rho_{j 0}\right)$ as we assume capacity increases with available bandwidth. By (6) and (8),

$$
u_{i j}^{\mathrm{ins}}>R_{j}^{\mathrm{ins}}-C_{j}^{\mathrm{ins}}\left(W_{j}+\Delta W_{i j}, \rho_{j 0}\right)=-R_{i}^{\mathrm{min}} .
$$

Taking the average, we get $R_{i}^{\min }>-u_{i j}^{\mathrm{f}}>0$. Similarly $R_{j}^{\min }>-u_{j i}^{\mathrm{f}}>0$. Therefore (20) holds, which implies the angle starting from $\overrightarrow{O D}$, going counterclockwise to $\overrightarrow{O F}$, is between 0 and $\pi$, i.e., $O \rightarrow D \rightarrow E \rightarrow F \rightarrow O$ is counterclockwise.

Proposition 3: If $u_{i j}^{\mathrm{f}} \geq 0$, then $S$ is on $E F$ and $P_{i j}^{\mathrm{c}}=1$.

Proof: Under the assumption, $E$ pareto dominates $D$, so $S$ is not on $O D, D E$ or $O F$. Therefore $S$ is on $E F$ as the result of mixing strategy profiles $\langle\mathrm{c}, \mathrm{c}\rangle$ and $\langle\mathrm{c}, \mathrm{n}\rangle$, which means the NBS strategy for $j$ is to always cooperate, i.e., $P_{i j}^{\mathrm{c}}=1$.

Next consider the case when $S$ coincides with $D$.

Proposition 4: $S=D$ if and only if

$$
\tan D>|\tan F| \text {. }
$$

Proof: (22) is necessary:

If $D=S, D$ is in the first quadrant, i.e., $u_{j i}^{\mathrm{f}} \geq 0$, so we must have $u_{i j}^{\mathrm{f}}<0$ by Proposition 3, i.e., $F$ is in the second quadrant. In this case, for $S=D$, we must have $\tan D>$ $-\tan F=|\tan F|$.

(22) is sufficient:

If $u_{i j}^{\mathrm{f}} \geq 0, F$ is in the first quadrant. Since (22) holds, $D$ is also in the first quadrant, i.e., $u_{j i}^{\mathrm{f}} \geq 0$, which leads to contradiction by Proposition 3. If $u_{j i}^{\mathrm{f}}<0, D$ is in the fourth quadrant and (22) is not possible. But when $u_{j i}^{\mathrm{f}} \geq 0>u_{i j}^{\mathrm{f}}$, $D$ is in the first quadrant and $F$ in the second quadrant. (22) implies $\tan D>-\tan F$ which in turn implies $S=D$.

The above proves that when (22) holds, $S=D$ and $P_{i j}^{\mathrm{c}}=$ 0 as in (12a). We also consider the sufficient and necessary condition for $S$ to lie in the interior of segment $D E$ (i.e., $S$ lies on $D E$ but does not coincide with $D$ or $E$ ), which corresponds to the case $0<P_{i j}^{\mathrm{c}}<1$ as a result of mixing the strategy profiles $\langle\mathrm{n}, \mathrm{c}\rangle$ and $\langle\mathrm{c}, \mathrm{c}\rangle$.

Proposition 5: $S$ is in the interior of $D E$ if and only if

$$
|\tan E|>|\tan F|>|\tan D| \text {. }
$$

Proof: (23) is necessary:

When $S \in D E$ but $S \neq D$ or $E$, the subgradient at $S$ coincides with $D E$ whose slope is $\tan F$. In this case, with Proposition 3, we must have $u_{i j}^{\mathrm{f}}<0$.

If $u_{j i}^{\mathrm{f}} \geq 0>u_{i j}^{\mathrm{f}}, D$ is in the first quadrant and $F$ in the second quadrant. If $E$ is in the first quadrant, for $S(\neq D$ or $E)$ to be on segment $D E$, we must have $\tan E>$ $-\tan F>\tan D$. If $E$ is in the second quadrant, we must have $-\tan F>\tan D$. Also, as $E, F$ are in the second quadrant, Proposition 2 implies $-\tan E>-\tan F$. In either case we have (23)

If $u_{i j}^{\mathrm{f}}<0$ and $u_{j i}^{\mathrm{f}}<0, D$ is in the fourth quadrant and $F$ is in the second quadrant. Proposition 2 implies $|\tan F|>$ $|\tan D|$. If $E$ is in the first quadrant, for $S$ to lie in the interior of $D E$, we must have $\tan E>-\tan F$. If $E$ is in the second quadrant, we must have $-\tan E>-\tan F$. Both cases imply $|\tan E|>|\tan F|$. Thus we again have (23).

(23) is sufficient:

When (23) holds, it is not possible that $u_{i j}^{\mathrm{f}}>0$ and $u_{j i}^{\mathrm{f}}>0$. Because in this case $D, E, F$ are all in the first quadrant and, as $\overrightarrow{O E}=\overrightarrow{O D}+\overrightarrow{O F}$, we must have

$$
\begin{aligned}
\tan E & =|\tan E| \\
& \leq \max (\tan F=|\tan F|, \tan D=|\tan D|),
\end{aligned}
$$


a contradiction to (23). It is not possible that $u_{i j}^{\mathrm{f}} \geq 0>u_{j i}^{\mathrm{f}}$ (i.e., $F$ is in the first quadrant and $D$ in the fourth quadrant), because if $E$ is in the first quadrant, we have $|\tan F|=$ $\tan F \geq \tan E=|\tan E|$ by Proposition 2, and if $E$ is in the fourth quadrant, $|\tan D|>|\tan E|$.

If $u_{j i}^{\mathrm{f}} \geq 0>u_{i j}^{\mathrm{f}}$, by checking out in which quadrant point $D, E, F$ can reside, (23) implies

$$
\begin{array}{cl}
\tan E>-\tan F>\tan D, & \text { if } \tan E>0, \\
-\tan F>\tan D, & \text { if } \tan E<0 .
\end{array}
$$

Both inequalities imply that $S$ is on segment $D E$.

If $u_{i j}^{\mathrm{f}}<0, u_{j i}^{\mathrm{f}}<0$, by checking out in which quadrant point $D, E, F$ can reside, (23) implies

$$
\begin{array}{cl}
\tan E>-\tan F, & \text { if } \tan E>0 \\
-\tan E>-\tan F, & \text { if } \tan E<0
\end{array}
$$

Both inequalities imply that $S$ is in the interior of $D E$.

The above proves that when (23) holds, $S$ is in the interior of $D E$, so we have

$$
\overrightarrow{O S}=P_{i j}^{\mathrm{c}} \cdot \overrightarrow{O E}+\left(1-P_{i j}^{\mathrm{c}}\right) \cdot \overrightarrow{O D} .
$$

Further, since $O D E F$ is a parallelogram and the horizontal image of $O S$ is a subgradient of $\mathcal{C}$ at $S$, it follows that $\tan S=$ $-\tan F$. Using this condition in (26) results in

$$
P_{i j}^{\mathrm{c}}=-\frac{P_{j i}}{2 P_{i j}}\left(\frac{R_{j}^{\mathrm{min}}}{u_{i j}^{\mathrm{f}}}+\frac{u_{j i}^{\mathrm{f}}}{R_{i}^{\mathrm{min}}}\right) .
$$

When $S \neq D$ and $S$ is not in the interior of $D E$, it follows $P_{i j}^{\mathrm{c}}=1$ as in $(12 \mathrm{c})$.

\section{REFERENCES}

[1] S. Mathur, L. Sankar, and N. Mandayam, "Coalitions in cooperative wireless networks," J. Sel. Areas Commun., vol. 26, pp. 1104-1115, Sep. 2008.

[2] S. Buchegger and J.-Y. Le Boudec, "Self-policing mobile ad hoc networks by reputation systems," IEEE Commun. Mag., vol. 43, pp. 101-107, July 2005.

[3] Q. He, D. Wu, and P. Khosla, "Sori: a secure and objective reputationbased incentive scheme for ad hoc networks," in Proc. IEEE Wireless Communications and Networks Conference (WCNC), Mar. 2004, pp. 825-830.

[4] Y. Liu and Y. R. Yang, "Reputation propagation and agreement in mobile ad-hoc networks," in Proc. IEEE Wireless Communications and Networks Conference (WCNC), vol. 3, Mar. 2003, pp. 1510-1515.

[5] S. Marti, T. Giuli, K. Lai, and M. Baker, "Mitigating routing misbehavior in mobile ad hoc networks," in Proc. Sixth International Conference on Mobile Computing and Networking, Aug. 2000, pp. 255-265.

[6] P. Michiardi and R. Molva, "Core: a cooperative reputation mechanism to enforce node cooperation in mobile ad hoc networks," in Communications and Multimedia Security Conference (CMS), 2002.

[7] L. Buttyan and J. P. Hubaux, "Stimulating cooperation in self-organizing mobile ad hoc networks," ACM J. Mobile Networks, vol. 8, pp. 579-592, Oct. 2003.

[8] S. Zhong, J. Chen, and Y. R. Yang, "Sprite: a simple, cheat proof, creditbased system for mobile ad-hoc networks," in Proc. IEEE INFOCOM, Apr. 2002, pp. 1987-1997.

[9] O. Ileri, S.-C. Mau, and N. Mandayam, "Pricing for enabling forwarding in self-configuring ad hoc networks," IEEE J. Sel. Areas Commun., vol. 23, pp. 151-162, Jan. 2005 .

[10] M. Lindstrom and P. Lungaro, "Resource delegation and rewards to stimulate forwarding in multihop cellular networks," in Proc. IEEE Vehicular Technology Conference (VTC), vol. 4, June 2005, pp. 21522156.

[11] L. Anderegg and S. Eidenbenz, "Ad hoc-VCG: a truthful and costefficient routing protocol for mobile ad hoc networks with selfish agents," in Proc. Mobicom, Apr. 2003, pp. 245-259.
[12] M. Felegyhazi, J. P. Hubaux, and L. Buttyan, "Nash equilibria of packet forwarding strategies in wireless ad hoc networks," IEEE Trans. Mobile Computing, vol. 5, pp. 463-475, May 2006.

[13] P. Michiardi and R. Molva, "Game theoretic analysis of security in mobile ad hoc networks," Institut Eurecom, Technical report, Apr. 2002.

[14] S. Zhong, L. E. Li, Y. Liu, and Y. R. Yang, "On designing incentive-compatible routing and forwarding protocols in wireless adhoc networks-an integrated approach using game theoretical and cryptographic techniques," in Proc. Mobicom, Apr. 2003, pp. 117-131.

[15] D. Zhang, O. Ileri, and N. Mandayam, "Bandwidth exchange as an incentive for relaying," in Proc. 42nd Annual Conference on Information Sciences and Systems (CISS), Princeton, NJ, Mar. 2008, pp. 749-754.

[16] Z. Zhang, J. Shi, H.-H. Chen, M. Guizani, and P. Qiu, "A cooperation strategy based on Nash Bargaining Solution in cooperative relay networks," IEEE Trans. Veh. Technol., vol. 57, no. 4, pp. 2570-2577, July 2008.

[17] H. Yin and S. Alamouti, "OFDMA: a broadband wireless access technology," in Proc. IEEE Sarnoff Symposium, 2006, pp. 1-4.

[18] "Draft standard for local and metropolitan area networks-part 16: air interface for fixed and mobile broadband wireless access systemsmultihop relay specification," IEEE, Unapproved Draft Std, Aug. 2007.

[19] "IEEE standard for local and metropolitan area networks part 16: air interface for fixed and mobile broadband wireless access systems amendment 2," IEEE Std 802.16e-2005 and IEEE Std 802.16-2004/Cor 1-2005, pp. 0_1-822, 2006.

[20] D. Fudenberg and J. Tirole, Game Theory. MIT Press, 1991.

[21] G. Owen, Game Theory, 3rd ed. Academic Press, 1995.

[22] "XOHM ExpressCard." [Online]. Available: http://www.xohm .com/en_US/shop/devices/pccard-samsung-swce100.html.

[23] "WiMAX Forum $\AA$ announces first certified MIMO $2.5 \mathrm{GHz}$ mobile WiMAX products," June 2008. [Online]. Available: http://www.wimaxforum.org/news/pr/view?item_key=cffca4e77e 1900b8fa727fe754a60be0db849e6.



Dan Zhang received the B.S. (Hons.) degree in 2000 and M.S. degree in 2005 from Shanghai Jiaotong University, all in electrical engineering. In 2005, he joined the Ph.D program of the ECE department at Rutgers University. Since 2007, he has been a graduate assistant of Prof. N. Mandayam at the Wireless Information Network Laboratory (WINLAB). His research interests include communication signal processing and wireless networking. $\mathrm{He}$ is also a M.S. candidate in Mathematics at Rutgers. Before he came to U.S.A., he worked for LSI Design \& Integration Corp. as a digital engineer, and for Micronas as a communication system engineer.

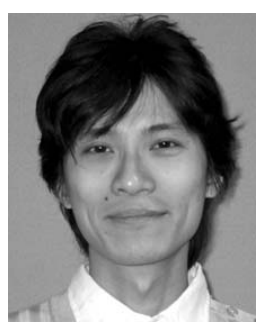

Ryoichi Shinkuma received the B.E., M.E., and Ph.D. degrees in Communications Engineering from Osaka University, Japan, in 2000, 2001, and 2003, respectively. In 2003, he joined the faculty of Communications and Computer Engineering, Graduate School of Informatics, Kyoto University, Japan, where he is currently an Assistant Professor. He was a Visiting Scholar at Wireless Information Network Laboratory (WINLAB), Rutgers, the State University of New Jersey, USA, from 2008 Fall to 2009 Fall. His recent research interests are in opportunistic communications, including data delivery in dynamic wireless networks, and control mechanisms for social communication networks. He is a recipient of Ericsson Young Scientist Award, Japan, in 2007. He is a member of IEICE, Japan. 


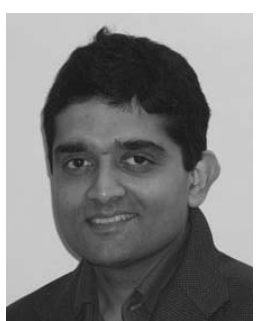

Narayan B. Mandayam received the B.Tech (Hons.) degree in 1989 from the Indian Institute of Technology, Kharagpur, and the M.S. and Ph.D. degrees in 1991 and 1994 from Rice University, all in electrical engineering. From 1994 to 1996, he was a Research Associate at the Wireless Information Network Laboratory (WINLAB), Rutgers University before joining the faculty of the Electrical and Computer Engineering department at Rutgers where he became Associate Professor in 2001 and Professor in 2003. Currently, he also serves as Associate Director at WINLAB. He was a visiting faculty fellow in the Department of Electrical Engineering, Princeton University in 2002 and a visiting faculty at the Indian Institute of Science in 2003. His research interests are in various aspects of wireless data transmission including system modeling and performance, signal processing and radio resource management with emphasis on techniques for cognitive radio networks.

Dr. Mandayam is a recipient of the Fred W. Ellersick Prize from the IEEE Communications Society in 2009 along with O. Ileri for their work on dynamic spectrum access models and spectrum policy. He is also a recipient of the Institute Silver Medal from the Indian Institute of Technology in 1989 and the National Science Foundation CAREER Award in 1998. He is a coauthor with C. Comaniciu and H. V. Poor of the book Wireless Networks: Multiuser Detection in Cross-Layer Design (Springer, NY). He has served as an Editor for the journals IEEE COMMUNICATION LETTERS and IEEE TRANSACTIONS ON WIRELESS COMMUNICATIONS. He has also served as a guest editor of the IEEE JSAC Special Issues on Adaptive, Spectrum Agile and Cognitive Radio Networks (2007) and Game Theory in Communication Systems (2008). He is a Fellow of the IEEE. 